\title{
Postprint
}

This is the accepted version of a paper published in Nanoscale.

This paper has been peer-reviewed but does not include the final publisher proofcorrections or journal pagination.

Citation for the original published paper (version of record):

A. Fairbrother, J. R. Sanchez-Valencia, B. Lauber, I. Shorubalko, P. Ruffieux, T. Hintermann, R. Fasel

High-vacuum synthesis and ambient stability of bottom-up graphene nanoribbons.

Nanoscale 9, 2785-2792 (2017)

https://doi.org/10.1039/C6NR08975E

Access to the published version may require subscription.

N.B. When citing this work, please cite the original published paper. 


\section{High vacuum synthesis and ambient stability of bottom-up graphene}

\section{nanoribbons}

Andrew Fairbrother, ${ }^{a}$ Juan-Ramon Sanchez-Valencia, ${ }^{a}$ Beat Lauber, ${ }^{a}$ Ivan Shorubalko, ${ }^{b}$ Pascal Ruffieux, ${ }^{a}$ Tobias Hintermann, ${ }^{c}$ Roman Fasel $^{\mathrm{a} *}$

ananotech@surfaces Laboratory, Empa, Swiss Federal Laboratories for Materials Science and Technology, 8600 Dübendorf, Switzerland

baboratory for Reliability Science and Technology, Empa, Swiss Federal Laboratories for Materials Science and Technology, 8600 Dübendorf, Switzerland

${ }^{c}$ BASF Schweiz AG, 4057 Basel, Switzerland

*E-mail: roman.fasel@empa.ch

Dr. A. Fairbrother current affiliation: Polymeric Materials Group, NIST, National Institute of Standards and Technology, Gaithersburg, MD 20899 USA

Dr. J.-R. Sanchez-Valencia current affiliation: Nanotechnology on Surfaces Lab, ICMS CSIC, Institute of Materials Science of Seville, 41092 Seville, Spain

Carbon-based nanomaterials such as graphene are at a crucial point in application development, and their promising potential which has been demonstrated at the laboratory scale must be translated to an industrial setting for commercialization. Graphene nanoribbons in particular overcome one limitation of graphene in some electronic applications because they exhibit a sizeable bandgap. However, synthesis of bottom-up graphene nanoribbons is most commonly performed under ultra-high vacuum conditions, which are costly and difficult to maintain in a manufacturing environment. Additionally, little is known about the stability of graphene nanoribbons under ambient conditions or during transfer to technologically relevant substrates and subsequent device processing. This work addresses some of these challenges, first by synthesizing bottom-up graphene nanoribbons under easily obtained high vacuum conditions and identifying water and oxygen as the residual gases responsible for interfering with proper coupling during the polymerization step. And second, by using Raman spectroscopy to probe the stability of nanoribbons during storage under ambient conditions, after transfer to arbitrary substrates, and after fabrication of field-effect transistor devices, which shows structurally intact nanoribbons even several months after synthesis. These findings demonstrate the potential of graphene nanoribbon technologies by addressing some limitations which might arise in their commercialization. 


\section{Introduction}

Graphene nanoribbons (GNRs) are narrow strips of graphene which have an appreciable bandgap due to lateral confinement, thus making them suitable for room temperature switching applications such as field-effect transistors $(\mathrm{FET}) .^{1-4}$ Aside from possessing a bandgap, these materials share many of the exceptional properties of graphene, including high thermal and electrical conductivity. GNRs synthesized via bottom-up approaches are especially suited to electronic device applications because narrower widths and precise edge topologies can be more easily obtained compared to the top-down synthesis of GNRs (i.e. lithography of graphene, unzipping of nanotubes). ${ }^{5-9}$ Furthermore, the atomic precision achieved by bottom-up synthesis allows one to deterministically define the magnitude of the bandgap. Bottom-up synthesis of GNRs is typically achieved through surface assisted coupling and cyclodehydrogenation of suitably selected precursor molecules, for example, 10,10'-dibromo-9,9'-bianthryl (DBBA) to form a 7 carbon atom wide, armchair edge ribbon (7AGNR) (Fig. 1a). ${ }^{10}$

In spite of appealing material properties, an array of production barriers must be overcome for commercialization, which include addressing the scalability, reproducibility, and robustness of a fabrication process, and in surmounting those barriers many promising new materials fail. Bottom-up GNR synthesis is almost exclusively performed under ultra-high vacuum conditions (UHV, $<10^{-9} \mathrm{mbar}$ ), which are not especially feasible for a high throughput manufacturing process due to the prohibitive costs for maintaining such an environment at a large scale. Two methods which are more suitable for industrial production of bottom-up GNRs include solution and high vacuum (HV, $10^{-6} \mathrm{mbar}$ ) processing. Solution-based synthesis is appealing because it is easily scalable for mass production, though it has shown limited success in producing extended and well-defined GNR structures. ${ }^{3,4,11-13}$ HV conditions are readily obtained in an industrial setting, and are already very common in the fields of thin film and semiconductor manufacturing. GNR synthesis under HV conditions is not widely studied, ${ }^{14}$ because UHV conditions are much more often employed in order to study the fundamental physical processes of on-surface synthesis. This is due to a reduced risk for contamination - the surface collision rate of air is $<10^{9}$ $\mathrm{cm}^{2} \cdot \mathrm{s}^{-1}$ at $10^{-11}$ mbar - which helps isolate and study surface chemical reactions with greater care and precision. ${ }^{15}$ The precursor coupling or polymerization step (Fig. 1a, middle molecule) is expected to be strongly influenced by the presence of extraneous molecules because they can react with the radical ends of a dehalogenated monomer or polymer, effectively terminating the coupling process or even leading to new, uncontrolled coupling motifs. Needless to say, for most applications the growth of well-defined and long ribbons is preferable to ill-defined and short ones.

The synthesis of GNRs using industrially viable methods addresses one of several bottlenecks for technological applications. Another potential impediment to their use is stability: GNRs must remain structurally intact over time and during 
subsequent device production processes, which may include exposure to various atmospheres and chemical environments. Due to the prevalence of silicon-based electronics, integration into a silicon manufacturing process is the most likely future scenario, in which case the GNRs may be exposed to heat, air, acids, solvents, etc. during the numerous fabrication steps. $^{1,16,17}$

In the framework of commercial viability, this work examines some of the potential constraints on GNR-technologies. First, GNRs have been synthesized under varying levels of vacuum and ribbon quality was ascertained by scanning tunneling microscopy (STM). A transition from ribbon-like to dendritic coupling occurs around a system base pressure of $10^{-6}$ mbar. At this point there is a critical partial pressure of water and oxygen, $\mathrm{p}_{\mathrm{H} 2 \mathrm{O}}{ }^{*} \mathrm{p}_{\mathrm{O} 2}=3 \times 10^{-15}$ mbar ${ }^{2}$, which is found to limit ribbon quality when present during the polymer formation step. Second, the stability of GNRs under ambient conditions has been investigated by Raman spectroscopy, which shows structurally intact ribbons even after several months of storage under ambient conditions. Raman measurements after post-synthesis transfer to semiconducting or insulating substrates and GNR-FET device fabrication show similar promise regarding GNR stability. Electrical measurements of back-gated GNR-FETs exhibit p-type semiconducting charge transport dominated by a large Schottky barrier, with a maximum on-current of 1 nA and $\mathrm{I}_{\mathrm{On}} / \mathrm{I}_{\mathrm{Off}}$ of $10^{3}$. The principle conclusions are that GNRs can in fact be synthesized under more industrially compatible HV conditions, not only under the UHV conditions used at the laboratory scale, and that GNRs are remarkably stable under potential processing environments.

\section{Results and Discussion}

\subsection{High vacuum GNR synthesis}

7-AGNRs are the model GNR system examined in this work because they are the first bottom-up GNRs to have been synthesized, and are thus far the most widely studied. ${ }^{\mathbf{1} 10,18}$ These are formed by surface-assisted coupling of $10,10^{\prime}$ dibromo-9,9'-bianthryl (DBBA, SYNTHON Chemicals GmbH) on a catalytic metal surface, followed by cyclodehydrogenation. A schematic of this reaction and the resulting GNRs are shown in Fig. 1a and $1 \mathrm{~b}$, with indicated temperatures for a Au(111) growth substrate. ${ }^{10}$ Under typical UHV conditions $\left(<10^{-9} \mathrm{mbar}\right)$ the deposition of the DBBA precursor and substrate heating described in the Experimental Section results in the growth of nanoribbons with an atomically precise width and edge morphology, as seen in Fig. 1c. ${ }^{10}$

The growth of 7-AGNRs under HV conditions is less well known, so next the base pressure was increased by three methods: 1) reducing pumping power, 2) pumping after venting, prior to system baking, or 3) dosing of water or oxygen into the chamber, as indicated in Fig. 1f. When the base pressure is increased up to $10^{-7}$ mbar GNR quality remains essentially unchanged, and length is not significantly decreased. The 7-AGNRs commonly exceed $40 \mathrm{~nm}$ in length, and there is no 
evidence of uncontrolled coupling. As the base pressure is increased slightly above $10^{-7} \mathrm{mbar}$ the characteristics of the GNRs are relatively unchanged in terms of edge quality, but ribbons are significantly shorter, now only occasionally exceeding $20 \mathrm{~nm}$. With further increase in the base pressure, up to about $4 \times 10^{-6} \mathrm{mbar}$, a transition from ribbon-like to dendritic coupling of DBBA occurs, and the resulting GNRs are much shorter, more poorly defined, and there is considerable cross-coupling (Fig. 1d). Here, and throughout this work, ribbon-like coupling or growth is defined as coupling along the back-bone of the DBBA precursor between dehalogenated carbon atoms (Fig. 1a), while dendritic coupling is defined as any alternative coupling other than the one intended from the precursor design. This dendritic growth is likely caused by a passivation of the $C$ radicals at the former bromine positions due to significant surface contamination. Above $10^{-6} \mathrm{mbar}$ entirely dendritic growth is observed, and no extended GNR segments are found (Fig. 1e).
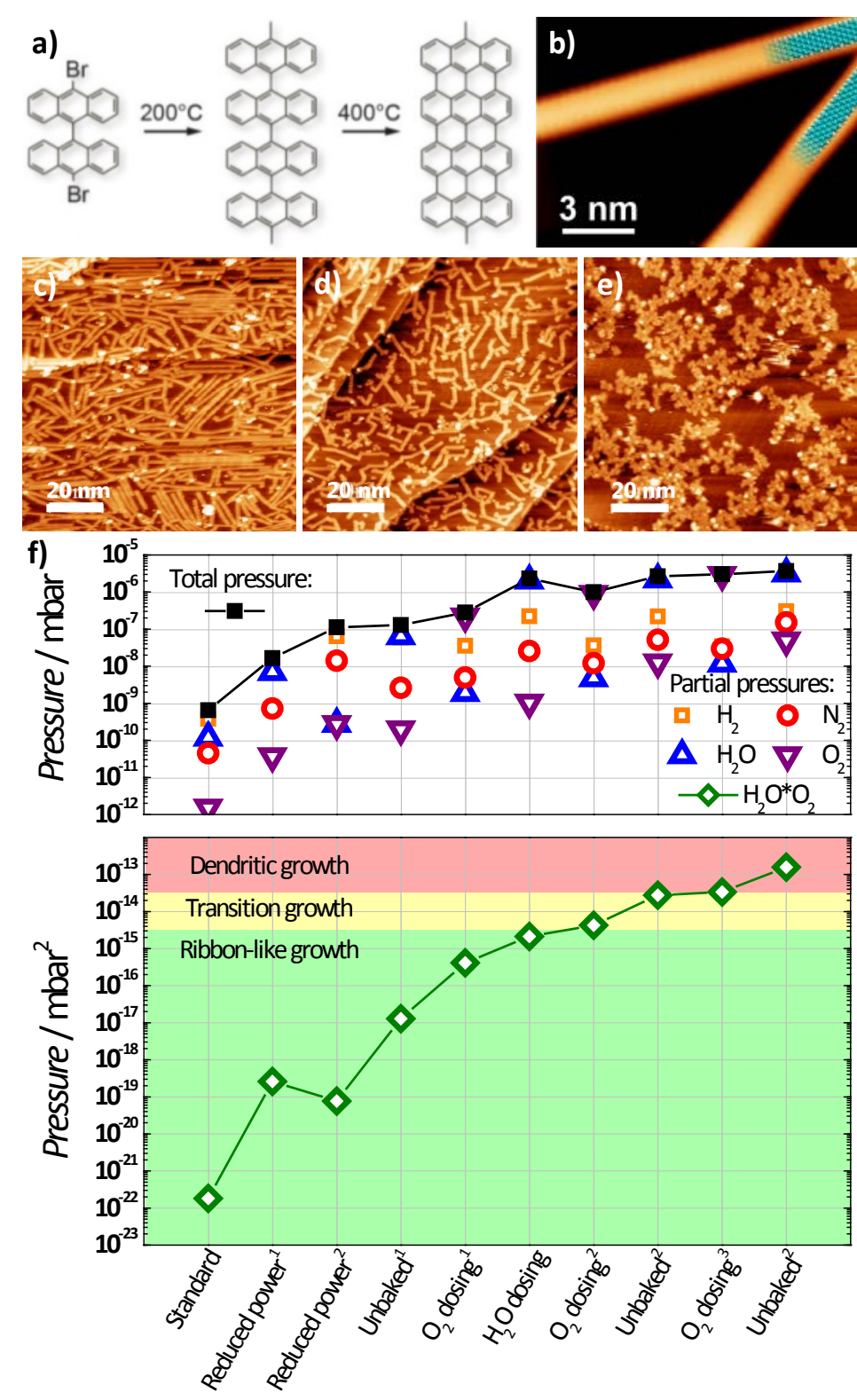
Fig. 1. Synthesis schematic for bottom-up 7-AGNRs on $\mathrm{Au}(111)$ showing the precursor, polymer, and ribbon states (a) and high resolution STM of the resulting GNRs with a molecular model overlaid (b). GNRs obtained from ribbon-like coupling (c), from transition growth (d), and from dendritic growth (e). The total system pressure and partial pressure of $\mathrm{H}_{2}, \mathrm{~N}_{2}, \mathrm{H}_{2} \mathrm{O}, \mathrm{O}_{2}$, and $\mathrm{H}_{2} \mathrm{O} * \mathrm{O}_{2}$ (f). The lower graph highlights the regimes under which the different GNR growth types are observed for different vacuum control conditions: standard - UHV system under normal operation with total pressure $<10^{-9} \mathrm{mbar}$; reduced power ${ }^{1}$ - turbomolecular pumping power reduced to $60 \%$; reduced power ${ }^{2}-$ main turbomolecular pump closed,

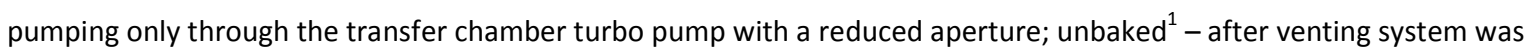

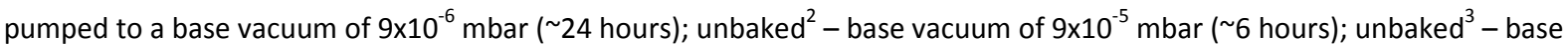
vacuum of $6 \times 10^{-5} \mathrm{mbar}\left(\sim 3\right.$ hours); $\mathrm{O}_{2}$ dosing ${ }^{1}$ - oxygen leaked into the chamber until a stable total pressure of $3 \times 10^{-7} \mathrm{mbar}$ was obtained; $\mathrm{O}_{2}$ dosing ${ }^{2}$ - total pressure of $9 \times 10^{-7} \mathrm{mbar} \mathrm{O}_{2}$ dosing ${ }^{3}$ - total pressure of $3 \times 10^{-6} \mathrm{mbar}$; and $\mathrm{H}_{2} \mathrm{O}$ dosing water leaked into chamber until a pressure of $2 \times 10^{-6} \mathrm{mbar}$ was obtained.

\subsubsection{Residual Gases During Synthesis}

There are a number of common residual gases in vacuum which contribute to the background pressure, including water $\left(\mathrm{H}_{2} \mathrm{O}\right)$, oxygen $\left(\mathrm{O}_{2}\right)$, carbon monoxide and dioxide $\left(\mathrm{CO}\right.$ and $\left.\mathrm{CO}_{2}\right)$, nitrogen $\left(\mathrm{N}_{2}\right)$, argon $(\mathrm{Ar})$, and hydrogen $\left(\mathrm{H}_{2}\right) .{ }^{15}$ Thus an examination of the base pressure alone as presented above provides an incomplete picture of what occurs during GNR synthesis at higher pressures. To better understand the influence of each of these gases they were monitored in-situ by means of a quadrupole mass spectrometer during the GNR synthesis process. Dosing of water in the chamber up to a partial pressure of $10^{-6}$ mbar resulted in proper GNR growth, while dosing of oxygen to this level and higher resulted in dendritic growth. However, when preparing GNRs in an unbaked system with a similar partial pressure of water, transition coupling was found to occur, while in the water dosing experiments ribbon-like growth occurred. From this it was determined that dendritic growth is not caused by a single residual gas, and in fact, a clear correlation between the product of partial pressures of water and oxygen is shown to predict the type of GNR coupling, as illustrated in the lower graph of Fig. 1f. Ribbon-like coupling occurs when $\mathrm{p}_{\mathrm{H} 2 \mathrm{O}} * \mathrm{p}_{\mathrm{O} 2}<3 \times 10^{-15} \mathrm{mbar}^{2}$, whereas dendritic growth occurs for $\mathrm{p}_{\mathrm{H} 2 \mathrm{O}} * \mathrm{p}_{\mathrm{O} 2}>3 \times 10^{-14}$ mbar $^{2}$. The mechanism for dendritic growth is not fully explored in this work, however, one possible mechanism may be the formation of hydroxyl groups from adsorbed $\mathrm{H}_{2} \mathrm{O}$ and $\mathrm{O}_{2}$ which can readily react with the radical ends of the dehalogenated carbon nanostructures. ${ }^{19-23}$ Defect sites in graphene-based materials can be especially reactive, and the carbon radicals on either end of the GNR polymer are essentially point defects. ${ }^{22,23}$ Further studies would be required to elucidate the specific pathway. It is worth highlighting that $\mathrm{Au}(111)$ has a lower dissociation rate for gases such as $\mathrm{H}_{2} \mathrm{O}, \mathrm{O}_{2}$, and $\mathrm{H}_{2}$ than other metals, which may make it especially suitable for high vacuum GNR synthesis. No clear correlations with growth type were found for the other gases measured $\left(\mathrm{H}_{2}\right.$ and $\left.\mathrm{N}_{2}\right)$. 


\subsubsection{Substrate Poisoning: Pre-Growth Contamination}

It is unclear from the above results exactly at what time the presence of residual gases inhibits ribbon formation: prior to coupling, or only during? One expectation already raised is that the influence of pressure will be significant during the coupling step of GNR synthesis, because at this time dehalogenation of the precursor molecules has already occurred, leaving radical ends on the monomers and polymer chains which can readily react with contaminants in the growth chamber. Once this occurs, the ribbon-like coupling of DBBA is effectively terminated, while unintended sites for coupling may even be formed. However, it is also possible that prior exposure to water and oxygen may essentially "poison" the catalytic growth surface and alter its reactivity, thus influencing the subsequent deposition and coupling of precursor molecules (for instance, other surfaces like $\mathrm{Cu}(111)$ have different reactivity which leads to other coupling motifs for the same DBBA molecule). ${ }^{24}$ To determine which is the actual case, water and oxygen were dosed into the growth chamber for 45 minutes with $\mathrm{p}_{\mathrm{H} 2 \mathrm{O}} * \mathrm{p}_{\mathrm{O} 2}=10^{-12} \mathrm{mbar}^{2}$, or in other words, far above the critical pressure previously identified to lead to dendritic coupling. During this time a clean growth substrate was held at either room temperature or $200{ }^{\circ} \mathrm{C}$ (i.e. the temperature during molecule deposition). Upon halting introduction of oxygen and water, then allowing pressure to recover below $10^{-8} \mathrm{mbar}$ ( 30 minutes), subsequent deposition of DBBA and annealing always results in proper ribbon-like coupling and GNR formation (Fig. S2a and S2b), which demonstrates that the presence of these gases before synthesis to poison the substrate has no significant effect on final ribbon quality, at least at the pressures identified as critical from the above UHV experiments. This clearly indicates that these gases interfere with GNR growth primarily when present during the coupling stage of synthesis, and that a substrate exposed to these gases is not responsible, at least at still relatively low pressure exposures used for this test $\left(\mathrm{p}_{\mathrm{H} 2 \mathrm{O}} * \mathrm{p}_{\mathrm{O} 2}=10^{-12} \mathrm{mbar}^{2}\right)$. The relatively low sticking coefficient of $\mathrm{Au}(111)$ means that it is possible or even likely that these gases desorb before deposition of DBBA. ${ }^{19,20}$ Nonetheless, it is useful to show what may occur under real vacuum conditions used for GNR growth.

As a final test for substrate poisoning, cleaned substrates were removed from the UHV chamber and exposed to atmospheric conditions for 45 minutes, and then reintroduced into the UHV chamber. This means that the substrates were exposed to a very high level of water and oxygen compared to the previous test, in addition to hydrocarbons and other atmospheric contaminants. ${ }^{15}$ The substrates were then scanned by STM at $40 \mathrm{~K}$ (Fig. S2c inset), and the presence of small, adsorbed molecules was identified. After proceeding with DBBA deposition and GNR synthesis as normal, dendritic growth was observed (Fig. S2C). Thus it is clear that while poisoning of the substrate at low partial pressures, even those identified as detrimental to ribbon growth in UHV, does not have a significant negative effect on ribbon quality, the exposure of substrates to atmospheric levels of water, oxygen, and other gases does inhibit proper ribbon growth. From these results it can be stated that the substrates must be cleaned in-situ under at least $\mathrm{HV}$ conditions (base pressure $<10^{-6}$ mbar) to allow GNR growth with proper coupling of the precursor molecules. 


\subsubsection{Kinetic Factors Influencing GNR Synthesis}

Up to this point water and oxygen have been identified as detrimental to proper coupling of GNR precursors, leading to dendritic coupling if present above a certain threshold $\left(\mathrm{p}_{\mathrm{H} 2 \mathrm{O}}{ }^{*} \mathrm{p}_{\mathrm{O} 2}>10^{-15} \mathrm{mbar}^{2}\right)$. Next, the presence of these gases has been found to be critical only when present during the coupling step, and that substrate poisoning (exposure before GNR synthesis) has no major effect, except in the case of extremely high exposure (atmospheric). However, in examining the surface chemical reactions there are kinetic factors in addition to the presence of chemical reactants which can also play a role in the coupling of the DBBA molecules. One such kinetic factor is the precursor molecule deposition rate, so to determine its effect it was decreased to $0.5 \AA ̊ / \mathrm{min}$, compared to $4 \AA$ A $/ \mathrm{min}$ in all previous results. Even under UHV conditions $\left(<10^{-9} \mathrm{mbar}\right)$, characteristics of transition growth are clearly seen at this lower deposition rate, similar to those seen in Fig. 1d. For this case kinetic factors can be attributed to the occurrence of transition growth: the longer residence time of DBBA molecules on the surface before reacting with other DBBA molecules means that there is also greater chance of reaction with water or oxygen. Thus the critical pressures of oxygen and water previously identified $\left(\mathrm{p}_{\mathrm{H} 2 \mathrm{O}} * \mathrm{p}_{\mathrm{O} 2}=10^{-15} \mathrm{mbar}^{2}\right)$ can be claimed as valid for a certain deposition rate $(4 \AA / \mathrm{min})$, while this pressure may in fact change by increasing or decreasing the deposition rate. Additionally, other factors not examined here may influence reaction kinetics, such as reaction temperatures and times, and the use of other substrates. ${ }^{24,25}$

\subsection{Structural Integrity of GNRs Over Time}

Achieving high vacuum synthesis of GNRs is a significant step forward for this technology and addresses the material quality issue which was expected to be a limiting factor under non-UHV synthesis conditions. Though moving beyond this, the material integrity of GNRs over time and during device processing becomes an important factor for their application. Raman spectroscopy was utilized to measure their stability in air, after transfer to arbitrary substrates, and after GNR-FET fabrication. The Raman spectrum of 7-AGNRs is characterized by a number of peaks common to all sp ${ }^{2}$-hybridized carbon materials like graphene, including the so-called G- $\left(-1590 \mathrm{~cm}^{-1}\right)$ and D-peaks $\left(-1350 \mathrm{~cm}^{-1}\right)$ and their overtones. ${ }^{10,26-29} \mathrm{GNR}$ edges are considered "defects" and contribute to the D-peak, so this is always present even in perfect GNRs. Additionally, GNRs exhibit C-H bending modes and a radial-breathing-like mode (RBLM) which is analogous to the radial-breathing mode in carbon nanotubes, and is a direct indicator of GNR width $\left(397 \mathrm{~cm}^{-1}\right.$ for the 7-AGNR). ${ }^{10,26,30,31}$

\subsubsection{Temporal Stability on Au/mica Growth Substrate}

7-AGNRs grown on Au/mica are found to have remarkable stability in air even after 8 months, as evidenced by the presence of narrow and intense characteristic peaks in Raman, including the RBLM, and a low D/G ratio (Fig. 2a). Compared with spectra recorded immediately after synthesis, over time there is a general trend of peak broadening, increase in the peak 
ratio of D/G, and a blue-shift which stabilizes after several days (Fig. 2b). STM of GNRs stored in air for seven days is shown in Fig. S3. It shows intact GNRs, with evidence of some adsorbates (atmospheric contamination). In spite of these changes, discussed next, the important result is that there are no significant structural modifications of the GNRs, as indicated by the persistence of the RBLM and other GNR related peaks over time.
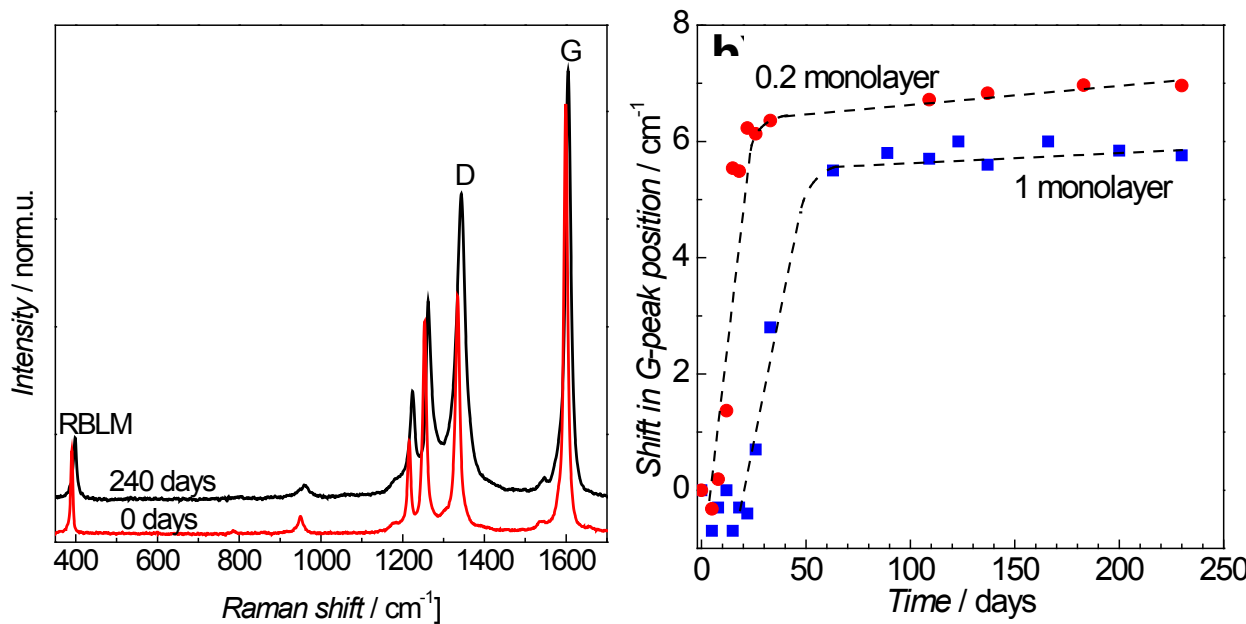

Fig. 2. Raman spectra of 7-AGNRs on Au/mica immediately after synthesis (red) and after 8 months (black) (normalized by G-peak intensity) (a). Time evolution of G-peak positions for low (0.2 monolayer) and high (1.0 monolayer) 7-AGNR coverage (b).

Exposure to air and the resulting adsorption and intercalation of airborne molecules is expected to be responsible for the changes observed over time, and three observations have been made which support this conclusion. First, the changes in Raman spectral features are not observed in GNRs which have been stored in UHV for over one month and then removed for measurement. Second, upon $200{ }^{\circ} \mathrm{C}$ vacuum heating of GNR samples stored in air for 5 months, the original positions of the Raman peaks are recovered. And third, the GNR coverage influences the time scale of the changes observed, in particular, the peak shifts are accelerated as the coverage is reduced. Dense GNR coverage can be expected to slow the intercalation of molecules by acting as a barrier to the substrate. The changes in spectral features - position and peak width - are expected due to an effective doping or compressive/tensile strain induced by airborne molecules. ${ }^{28,32-34}$ In both cases there is a distortion of the GNR lattice, which modifies bond lengths and leads to peak shift and broadening.

\subsubsection{Post-Transfer and FET Processing Stability}

For practically all device applications GNRs must be transferred from their metallic growth substrate, in this instance 200 $\mathrm{nm}-\mathrm{Au}(111) / \mathrm{mica}$, onto an electrically semiconducting or insulating substrate in order to fully exploit their exceptional material properties. ${ }^{1,35,36}$ In fact, the stability of GNRs on other substrates is arguably more important than stability on the 
growth substrate, because in a manufacturing process the GNRs would be transferred shortly after synthesis in order to fabricate devices. In this work a non-membrane based method is utilized, as previously described in Ref. ${ }^{18}$. The method has been modified by replacing $\mathrm{HF}$ with $\mathrm{HCl}$, a much safer alternative for processing. The transfer process onto $\mathrm{SiO}_{2} / \mathrm{Si}$ results in a near simultaneous shift in the Raman peaks of the GNRs, which is approximately of the same order of magnitude as seen for GNRs on Au stored in air during 8 months, and the intensity ratio of D/G increases from about 0.4 to 0.9 (Fig. 3a) (for Raman after transfer to $\mathrm{Al}_{2} \mathrm{O}_{3}$ and $\mathrm{CaF}_{2}$ see Fig. S4). These changes are observed in other transfer processes (both membrane and non-membrane), ${ }^{1,35,36}$ and are expected to be a result of removal of the growth substrate and consequent differences in lattice strain, as opposed to modifications induced by the particular process used here. In fact, studies on decoupled GNRs show that the substrate can influence material properties. ${ }^{25,37}$ In GNRs the D-peak is related to the intrinsic presence of edges, as opposed to other defects, and these edges will interact more or less strongly with the environment (e.g. the underlying substrate surface or the liquid environment), which may change the $D / G$ ratio correspondingly. A related observation comes from GNRs prepared in solution, in contrast to a metal surface. Those GNRs have a relatively high D/G ratio after synthesis, because they do not exhibit a strong substrate interaction. ${ }^{3,11-13}$ After two to three days the positions and relative intensities of all peaks reach a plateau similar to that seen for GNRs/Au, and they remain unchanged for at least one month, the time frame during which they were studied.
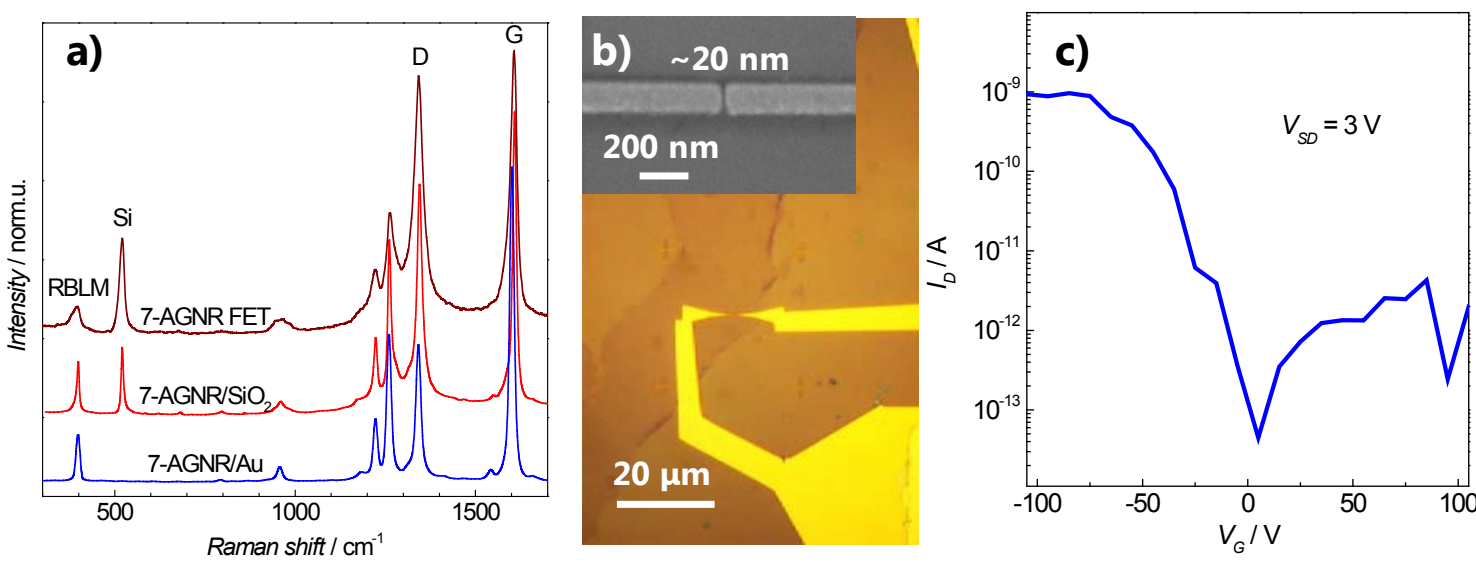

Fig. 3. Raman spectra of 7-AGNRs on $\mathrm{Au} /$ mica, after transfer to $\mathrm{SiO}_{2} / \mathrm{Si}$, and after GNR-FET processing (a). Optical micrograph showing a back-gated GNR-FET, inset shows a SEM image of the $20 \mathrm{~nm}$ gap between the source and drain electrodes (b). Current-voltage characteristics of a 7-AGNR-FET showing p-type semiconducting charge transport (c).

As a final test of the robustness of 7-AGNRs for potential applications, back-gated GNR-FETs were fabricated from GNRs transferred onto $\mathrm{SiO}_{2} / \mathrm{Si}$. This was to determine if they could be feasibly integrated into pre-existing production lines with other electronic materials. This was done by using e-beam lithography to define the source and drain contacts with a $20 \mathrm{~nm}$ gap (Fig. 3b), followed by metallization and lift-off, described in the Experimental description. Raman measurements 
performed after these processes show intact GNRs, though with wider peaks than just after transfer. This is attributed mainly to the time required to fully complete FET fabrication, though most critically, the persistence of the characteristic GNR peaks indicates that no significant structural alteration has occurred during device processing. Electrical characterization of a 7-AGNR-FET in Fig. 3c shows p-type semiconducting charge transport with a maximum $\mathrm{I}_{\mathrm{On}}$ of $1 \mathrm{nA}$ at $\mathrm{V}_{\mathrm{SD}}=3 \mathrm{~V}$, and $\mathrm{I}_{\mathrm{On}} / \mathrm{I}_{\mathrm{Off}}$ of $10^{3}$ at a gate voltage of $-105 \mathrm{~V}$ (additional data shown in Fig. S5). The observed $\mathrm{I}_{\text {off }}$ is induced by gate leakage, and noise in the current is probably due to rearrangement of charge traps in the $\mathrm{SiO}_{2} / \mathrm{Si}$ substrate. These results are similar to previous work on 7-AGNR-FETs, ${ }^{1}$ and one of the main conclusions is that charge transport is dominated by a large Schottky barrier due to the large band gap of the 7-AGNR (3.8 eV for a freestanding ribbon)..$^{38}$ In spite of the non-ideal band gap for a FET, the observed transport and Raman measurements indicate conclusively that the 7-AGNRs have not been destroyed after being subjected to numerous nano/microelectronic fabrication processes. Additional tests on a "blank" FET fabricated on bare $\mathrm{SiO}_{2} / \mathrm{Si}$ and a 7-AGNR-FET with a $1 \mu \mathrm{m}$ source-drain gap do not show any conduction, indicating that the observed characteristics originate from individual GNRs bridging the $20 \mathrm{~nm}$ electrode gap, and not from substrate or inter-ribbon conduction.

\section{Conclusion}

This work has addressed open questions about GNR-based technologies, in particular on the feasibility of production scaling and material stability. First, the possibility of high vacuum $\left(10^{-6} \mathrm{mbar}\right)$ synthesis of 7-AGNRs was demonstrated. The presence of water and oxygen above a critical pressure $\left(\mathrm{p}_{\mathrm{H} 2 \mathrm{O}} \mathrm{p}_{\mathrm{O} 2}=10^{-15} \mathrm{mbar}^{2}\right)$ was shown to alter the coupling step of the GNR precursor (DBBA), leading to growth of dendritic, rather than ribbon structures. Second, 7-AGNRs on gold were found to have remarkable stability under ambient conditions for several months, and also after transfer to arbitrary substrates. Raman spectroscopy indicates that the GNRs remain structurally intact over this period, with changes in peak position and width due to adsorption or intercalation of atmospheric contaminants. The robustness of the GNRs was also demonstrated after GNR-FET fabrication, in which semiconducting charge transport was measured. These results serve as a preliminary demonstration of the potential of GNRs in a commercial manufacturing setting, and warrant continued research.

\section{Experimental Section}

\subsection{GNR Synthesis}

7-AGNRs are the model GNR system examined in this work because they are the first bottom-up GNRs to have been synthesized, and are thus far the most widely studied. ${ }^{1,10,18}$ These are formed by surface-assisted coupling of $10,10^{\prime}$ dibromo-9,9'-bianthryl (DBBA, SYNTHON Chemicals GmbH, purified by recrystallization) on a catalytic metal surface, followed by cyclodehydrogenation. A schematic of this reaction and the resulting GNRs are shown in Fig. 1a and $1 \mathrm{~b}$, where the indicated temperatures are for a $\mathrm{Au}(111)$ growth substrate. In this work the substrate is $200 \mathrm{~nm}-\mathrm{Au}(111) / \mathrm{mica}$ (Phasis), 
which is first subjected to two cleaning cycles including $1 \mathrm{kV} \mathrm{Ar}{ }^{+}$sputtering and degassing/annealing at $470{ }^{\circ} \mathrm{C}$ in UHV. DBBA molecules are then sublimed $\left(\sim 200^{\circ} \mathrm{C}, 4-5 \AA / \mathrm{min}\right)$ for $1-4$ minutes onto the substrate which is held at $200{ }^{\circ} \mathrm{C}$, depending on the desired GNR coverage. After deposition, the temperature is held at $200{ }^{\circ} \mathrm{C}$ for ten minutes to induce dehalogenation of the precursor and subsequent $\mathrm{C}-\mathrm{C}$ coupling to form the GNR polymer, and then increased to $400{ }^{\circ} \mathrm{C}$ and held for ten minutes to cyclodehydrogenate the polymer and form planarized ribbons.

In the first part of this work the background pressure was varied to determine the threshold base pressure necessary for GNR synthesis. To control the pressure various means were employed, including reducing power of the turbomolecular pump $(60 \%$, and using only the transfer chamber turbo), using a chamber prior to bake-out (a procedure used to desorb water and other gases from the chamber walls and achieve UHV conditions) and pumped for a limited time ( 24, 6, and 3 hours), and intentional dosing of gases (water, oxygen) into the preparation chamber to obtain a particular base pressure. For in-situ characterization of the ribbon growth conditions a mass spectrometer (Pfeiffer QMA 200) was attached directly to the vacuum chamber to measure partial pressure of gases. Scanning tunneling microscopy (Omicron VT-STM) was used to characterize the ribbons under the various growth conditions. Measurements were performed at room temperature with typical scanning parameters including -1 V tunnel voltage, and 20-30 pA tunnel current in constant current mode.

\subsection{GNR Stability, Transfer, and FET Fabrication}

In the second part, the stability of GNRs was examined under different conditions. GNRs were stored in the dark $\left(\sim 25^{\circ} \mathrm{C}\right.$, $\sim 30-60 \%$ relative humidity) and periodically removed for Raman spectroscopy measurements over a period of 8 months. These were made with a Bruker SENTERRA Raman Microscope using a $532 \mathrm{~nm}$ diode laser with $2 \mathrm{~mW}$ power and 50x objective lens, resulting in a 1-2 $\mu \mathrm{m}$ spot size. No thermal effects were identified under these measurement conditions. An average of four spectra from different points was taken for each sample measurement.

GNRs were also transferred from their growth substrate (200 nm-Au/mica) to technologically relevant substrates (i.e. semiconducting or insulating, $285 \mathrm{~nm}-\mathrm{SiO}_{2} / \mathrm{p}++\mathrm{Si}, \mathrm{Al}_{2} \mathrm{O}_{3}$, and $\mathrm{CaF}_{2}(\mathrm{NOVA}$ Electronic Materials and Crystal $\left.\mathrm{GmbH})\right)$ and a similar time-dependent study was made. A non-membrane transfer was utilized, which involves cleavage of the GNR/Au film from mica by floating on hydrochloric acid, placing the GNR/Au stack onto an arbitrary substrate, and then etching away the Au film. ${ }^{18}$

After GNR transfer, back-gated GNR-FETs were fabricated on $\mathrm{GNR} / \mathrm{SiO}_{2} / \mathrm{Si}$ using electron beam lithography to define source and drain electrodes with a $20 \mathrm{~nm}$ gap, followed by metallization and lift-off. A double-layer resist was used to create the undercut cross-section needed for lift-off. The first layer was 50K PMMA/chlorobenze (AR-P 631.04, Allresist), which leaves 
no residues after development, and thus does not inhibit direct contact between the evaporated metal and GNRs. The second layer was diluted 950K PMMA/chlorobenzene (AR-P 671.04, Allresist), which has higher contrast and clearance dose than the 50K PMMA. After e-beam exposure (Raith 150) development was done in a solution of MIBK:IPA (1:3). 20 nm gaps can be reliably and reproducibly obtained with such a resist sequence. Next a $1 \mathrm{~nm}$ thick wetting layer of chromium, and then a $20 \mathrm{~nm}$ think layer of gold were deposited using e-beam evaporation (Evaporation Plassys II). The final step is a standard lift-off process with acetone. The resulting source-drain electrode configuration is shown in the inset of Fig. $3 \mathrm{~b}$.

Electrical characterization was performed at room temperature in air using standard Keithley source-measure units. A source-drain voltage $\left(\mathrm{V}_{\mathrm{SD}}\right)$ was applied between the electrodes, and the gate voltage $\left(\mathrm{V}_{\mathrm{G}}\right)$ was applied to the Si substrate, then corresponding $\mathrm{I}_{\mathrm{D}}$ and $\mathrm{I}_{\mathrm{G}}$ were measured. All GNR-FET structures showed qualitatively similar electrical characteristics, as shown in Fig. 3c. To demonstrate that charge transport occurs through individual GNRs, two additional device structures were fabricated and then tested. First, the $20 \mathrm{~nm}$ gap electrode geometry was made on a bare $\mathrm{SiO}_{2} / \mathrm{Si}_{\mathrm{s}} \mathrm{substrate}$. This "blank" device showed no conductance. Second, the source-drain configuration was changed to form a $1 \mu \mathrm{m}$ gap on the transferred GNR area. This device also showed no conductance, which indicates that inter-ribbon charge transfer is below detection limits. Both of these tests indicate that the electrical characteristics presented in Fig. 3c originate from individual GNRs bridging the electrode gaps.

\section{Supplementary Information}

Supplementary Information is available online from the publisher.

\section{Acknowledgements}

A.F. and J.R.S.V. contributed equally to this work. The authors would like to thank Dr. Andreas Borgschulte for use of the Raman spectrometer, Lukas Eisenring and Lukas Rotach for technical support of the UHV systems, and staff at the ETH Zurich FIRST lab for maintenance and assistance with equipment for device fabrication. Financial support was provided by the Swiss National Science Foundation, the Office of Naval Research BRC program, and the European Commission Graphene Flagship.

1 P. B. Bennett, Z. Pedramrazi, A. Madani, Y.-C. Chen, D. G. de Oteyza, C. Chen, F. R. Fischer, M. F. Crommie and J. Bokor, Appl. Phys. Lett., 2013, 103, 253114.

2 W. S. Hwang, P. Zhao, K. Tahy, L. O. Nyakiti, V. D. Wheeler, R. L. Myers-Ward, C. R. E. Jr, D. K. Gaskill, J. A. Robinson, W. Haensch, H. (Grace) Xing, A. Seabaugh and D. Jena, APL Mater., 2015, 3, 011101.

3 U. Zschieschang, H. Klauk, I. B. Müeller, A. J. Strudwick, T. Hintermann, M. G. Schwab, A. Narita, X. Feng, K. Müellen and R. T. Weitz, Adv. Electron. Mater., 2015, 1, n/a-n/a.

4 J. Gao, F. J. Uribe-Romo, J. D. Saathoff, H. Arslan, C. R. Crick, S. J. Hein, B. Itin, P. Clancy, W. R. Dichtel and Y.-L. Loo, ACS Nano, 2016, 10, 4847-4856.

5 Z. Chen, Y.-M. Lin, M. J. Rooks and P. Avouris, Phys. E Low-Dimens. Syst. Nanostructures, 2007, 40, $228-232$. 
6 X. Wang, Y. Ouyang, X. Li, H. Wang, J. Guo and H. Dai, Phys. Rev. Lett., 2008, 100.

7 D. V. Kosynkin, A. L. Higginbotham, A. Sinitskii, J. R. Lomeda, A. Dimiev, B. K. Price and J. M. Tour, Nature, 2009, 458, 872-876.

8 L. Jiao, L. Zhang, X. Wang, G. Diankov and H. Dai, Nature, 2009, 458, 877-880.

9 J. Feng, W. Li, X. Qian, J. Qi, L. Qi and J. Li, Nanoscale, 2012, 4, 4883-4899.

10 J. Cai, P. Ruffieux, R. Jaafar, M. Bieri, T. Braun, S. Blankenburg, M. Muoth, A. P. Seitsonen, M. Saleh, X. Feng, K. Müllen and R. Fasel, Nature, 2010, 466, 470-473.

11 T. H. Vo, M. Shekhirev, D. A. Kunkel, M. D. Morton, E. Berglund, L. Kong, P. M. Wilson, P. A. Dowben, A. Enders and A. Sinitskii, Nat. Commun., 2014, 5, 3189.

12 A. Narita, X. Feng, Y. Hernandez, S. A. Jensen, M. Bonn, H. Yang, I. A. Verzhbitskiy, C. Casiraghi, M. R. Hansen, A. H. R. Koch, G. Fytas, O. Ivasenko, B. Li, K. S. Mali, T. Balandina, S. Mahesh, S. De Feyter and K. Müllen, Nat. Chem., 2014, 6, 126-132.

13 A. N. Abbas, G. Liu, A. Narita, M. Orosco, X. Feng, K. Müllen and C. Zhou, J. Am. Chem. Soc., 2014, 136, 7555-7558.

14 H. Sakaguchi, Y. Kawagoe, Y. Hirano, T. Iruka, M. Yano and T. Nakae, Adv. Mater., 2014, 26, 4134-4138.

15 D. Hoffman, B. Singh and J. H. Thomas III, Handbook of Vacuum Science and Technology, Academic Press, 1st edn., 1997.

16 J. A. Robinson, M. LaBella, M. Zhu, M. Hollander, R. Kasarda, Z. Hughes, K. Trumbull, R. Cavalero and D. Snyder, Appl. Phys. Lett., 2011, 98, 053103.

17 K. Kim, J.-Y. Choi, T. Kim, S.-H. Cho and H.-J. Chung, Nature, 2011, 479, 338-344.

18 J. Cai, C. A. Pignedoli, L. Talirz, P. Ruffieux, H. Söde, L. Liang, V. Meunier, R. Berger, R. Li, X. Feng, K. Müllen and R. Fasel, Nat. Nanotechnol., 2014, 9, 896-900.

19 M. A. Henderson, Surf. Sci. Rep., 2002, 46, 1-308.

20 A. Hodgson and S. Haq, Surf. Sci. Rep., 2009, 64, 381-451.

21 E. M. Stuve and R. J. Madix, Surf. Sci., 1981, 111, 11-25.

22 N. Ghaderi and M. Peressi, J. Phys. Chem. C, 2010, 114, 21625-21630.

23 F. Banhart, J. Kotakoski and A. V. Krasheninnikov, ACS Nano, 2011, 5, 26-41.

24 C. Sánchez-Sánchez, T. Dienel, O. Deniz, P. Ruffieux, R. Berger, X. Feng, K. Müllen and R. Fasel, ACS Nano, 2016, 10, 8006-8011.

25 S. Wang, L. Talirz, C. A. Pignedoli, X. Feng, K. Müllen, R. Fasel and P. Ruffieux, Nat. Commun., 2016, 7, 11507.

26 R. Gillen, M. Mohr and J. Maultzsch, Phys. Rev. B, 2010, 81.

27 R. Saito, M. Furukawa, G. Dresselhaus and M. S. Dresselhaus, J. Phys. Condens. Matter, 2010, 22, 334203.

28 Z. H. Ni, T. Yu, Y. H. Lu, Y. Y. Wang, Y. P. Feng and Z. X. Shen, ACS Nano, 2008, 2, 2301-2305.

29 X. Jia, J. Campos-Delgado, M. Terrones, V. Meunier and M. S. Dresselhaus, Nanoscale, 2011, 3, 86-95.

30 R. Peköz, X. Feng and D. Donadio, J. Phys. Condens. Matter, 2012, 24, 104023.

31 I. A. Verzhbitskiy, M. D. Corato, A. Ruini, E. Molinari, A. Narita, Y. Hu, M. G. Schwab, M. Bruna, D. Yoon, S. Milana, X. Feng, K. Müllen, A. C. Ferrari, C. Casiraghi and D. Prezzi, Nano Lett., 2016, 16, 3442-3447.

32 J.-U. Lee, D. Yoon and H. Cheong, Nano Lett., 2012, 12, 4444-4448.

33 S. Ryu, J. Maultzsch, M. Y. Han, P. Kim and L. E. Brus, ACS Nano, 2011, 5, 4123-4130.

34 J. Zabel, R. R. Nair, A. Ott, T. Georgiou, A. K. Geim, K. S. Novoselov and C. Casiraghi, Nano Lett., 2012, 12, 617-621.

35 D. Q. McNerny, B. Viswanath, D. Copic, F. R. Laye, C. Prohoda, A. C. Brieland-Shoultz, E. S. Polsen, N. T. Dee, V. S.

Veerasamy and A. J. Hart, Sci. Rep., 2014, 4.

36 J. Kang, D. Shin, S. Bae and B. H. Hong, Nanoscale, 2012, 4, 5527.

37 J. Repp and G. Meyer, Appl. Phys. -Mater. Sci. Process., 2006, 85, 399-406.

38 L. Yang, C.-H. Park, Y.-W. Son, M. L. Cohen and S. G. Louie, Phys. Rev. Lett., 2007, 99, 186801. 